\title{
Uncertain Reasoning of Distribution Network Planning for Distributed Generator
}

\author{
Jiong Yan $^{1, *}$ and Fei Wang ${ }^{2}$ \\ ${ }^{1}$ State Grid Hubei Economic Research Institute, Wuhan, Hubei 430077, China \\ ${ }^{2}$ Department of Electronics and Information Engineering, College of Physical Science and Technology, Central China Normal \\ University, Wuhan, Hubei 430079, China \\ ${ }^{*}$ Corresponding author
}

\begin{abstract}
In order to study the impact of distributed generation (DG) in distribution network planning, a fuzzy programming approach is proposed. Triangular fuzzy numbers are used to describe the uncertainty of DG. Based on credibility theory, the network planning model is established. Fuzzy power flow is calculated, and the fitness function is determined based on the power flow results, and genetic algorithm (GA) is proposed to solve the model. The simulation results of the proposed approach are compared with other planning methods, the effectiveness of the propose approach is proved.
\end{abstract}

Keywords-distribution network; network planning; distributed generation; uncertainty; fuzzy

\section{INTRODUCTION}

Power system distribution network not only plays a key role for quality and reliability of electricity supply, but also can relieve environment crisis[1]. Distributed Generation (DG) access to the normal operation of the distribution network had a significant impact on increasing the distribution network planning difficulties and uncertainties, to the distribution network operation and planning challenges[2].

Reasonable network planning not only supply right direction of the development of the power grid, but also help reduce future retrofit in future and reduce fixed investment from power company[3]. Generally, distribution network structure containing DG planning is a multi-objective and multi-constraint optimization problem, so it should be considered all kinds of uncertainties in order to meet the actual requirements[4]. Usually, fuzzy analysis, stochastic theory, interval number optimization algorithms and scene analysis method are applied to deal with these uncertainties in distribution network planning. Based on fuzzy phenomena credibility theory, Ref.[5] introduces flow calculation, fuzzy power flow algorithm. A distribution network planning is carried out by constructing a fuzzy modeling capacity factor trapezoidal membership function of wind power in [6]. By taking into account load forecasting value and some types of DG output power uncertainty construct fuzzy expected value model in [7]. In this paper, by considering the influence of uncertainty into account DG, an adaptive Chaos Particle Swarm Optimization (ACPSO) output for DG modeling is constructed, then distribution network structure planning model based on fuzzy credibility theory is discussed, and a distributed power optimization planning is given.

\section{The Model of Distribution Network Planning}

DG with uncertainties of output power mainly includes photovoltaic power and wind power. There are two main construction modes: one is from the power company, the other is from user-DG. Generally speaking, DG building by Electricity Company usually designs its location and capacity based on climate, geography, load level planning area and other factors, so output power of the DG is usually certainly. Conversely, user-DG usually involves all kinds of different requirements, so it contains uncertainties which are difficult to describe. So these two situations should be modeled separately in the process of planning for DG.

In order to take advantage of renewable energy, consider the following model

$$
\mathrm{F}_{\mathrm{obj}}=\max \sum_{t=1}^{\mathrm{T} / \Delta t}\left(\sum_{i=1}^{N_{b}}\left(\sum_{j=1}^{N_{\text {sppe }}}\left(n_{i j}^{d g} \times p_{j}^{d g} \times \eta_{j}^{d g}(t)\right)\right) \times \Delta t\right)
$$

where $\mathrm{T}$ is the total number of hours, $\Delta t$ is Each time duration in hours, here $\Delta t$ is $1 \mathrm{~h}$; $\mathrm{t}$ is the number of periods., $N_{b}$ is the number of nodes, $N_{\text {type }}$ is the number of types to be elected as DG, $n_{i j}^{d g}$ is the number of DG node of the type of installation $\mathrm{j}, p_{j}^{\mathrm{dg}}$ is the rated power of every $\mathrm{j}$ type, $\eta_{j}^{\mathrm{dg}}(t)$ is the percentage of rated power with $\mathrm{j}$ type.

\section{A. Power Balance Constraint}

The equality constraint of the DG can be expressed as

$$
\left\{\begin{array}{l}
P_{G i}(t)+\sum_{j=1}^{N_{t y p e}}\left(n_{i j}^{d g} \times P_{d g j} \times \eta_{j}^{d g}(t)\right)-\eta^{t}(t) \times P_{L_{i}}=U_{i}(t) \sum_{j \in i} U_{j}(t) \times\left(G_{i j} \cos \theta_{i j}+B_{i j} \sin \theta_{i j}\right) \\
Q_{G i}(t)+\sum_{j=1}^{N_{t y p e}}\left(n_{i j}^{d g} \times P_{d g j} \times \eta_{j}^{d g}(t)\right) \times \tan \alpha-\eta^{L}(t) \times Q_{L_{i}}=U_{i}(t) \sum_{j \in l} U_{j}(t) \times\left(G_{i j} \cos \theta_{i j}+B_{i j} \sin \theta_{i j}\right)
\end{array}\right.
$$

where $P_{G i}(t)$ is node $i$ generator active power output in the period $t, N_{\text {type }}$ is the type of DG; $\eta_{j}^{d g}(t)$ is the ratio of the 
output power from $\mathrm{j}$ type at time intervals, $n_{i j}^{d g}$ is the number of DG node with the type; $P_{d g j}$ is the rated power of type $j$, $\eta^{L}(t)$ is load ratio at time intervals, $P_{L_{i}}$ is the maximum power load of $\mathrm{I}, U_{i}(t)$ is i node amplitude of voltage vector period $\mathrm{t} ; G_{i j}$ and $B_{i j}$ are the real part and imaginary part of the matrix, respectively; $\theta_{i j}$ represents phase angle difference between i and j node; $Q_{G i}(t)$ is wasted effort of the generator in the period t, $\alpha$ is power factor angle, $Q_{L_{i}}$ is the maximum wasted effort of node $i$.

\section{B. Branch Flow Constraints}

$$
S_{i}(t) \leq S_{i \max }
$$

where $S_{i \max }$ is branch i transmit power limit, it is desirable thermal stability limit line, $S_{i}(t)$ is in the period $t$ to the branch of the transmission power, the specific formula as:

$$
S_{i}(t)=\sqrt{P_{i}(t)^{2}+Q_{i}(t)^{2}}
$$

where $P_{i}(t)$ and $Q_{i}(t)$ is the first segment of branch node i in the period $t$ of active and reactive power through.

\section{The Voltage Constraint of Nodes}

$20 \mathrm{kV}$ and below the three-phase supply voltage deviation is $7 \%$ of the standard voltage.

For $10 \mathrm{kV}$ voltage distribution network, $7 \%$ of deviation limitation of the limit value of node is chosen to satisfy the following relationship:

$$
0.93 U_{N} \leq U_{i}(t) \leq 1.07 U_{N}\left(i=1 \ldots N_{b}\right)
$$

where $U_{N}$ is the nominal voltage, $U_{i}(t)$ is voltage amplitude of node $i$, and $N_{b}$ is the number of nodes.

\section{The Installed Capacity Constraints of DG}

Since FAN and PV have accompanied and volatility, too much access will affect the power quality and operational stability, it is necessary to limit the DG capacity as follows

$$
\begin{array}{cc}
G_{w g i} \leq G_{w g \max }, & i \in N_{d g} \\
G_{p v i} \leq G_{p v \max }, & i \in N_{d g}
\end{array}
$$

$$
\sum_{i}^{N_{d g}}\left(G_{w g i}+G_{p v i}\right) \leq G_{\max }, \quad i \in N_{d g}
$$

where $G_{w g i}$ and $G_{p v i}$ is the capacity of FAN and PV which access node I and $G_{\max }$ is capacity limit allow of DG which access to the distribution system, $N_{d g}$ is DG candidate node set.

\section{AdAPtive ChaOs PARTICle SWARM Optimization}

Although the particle swarm optimizationl algorithm (PSO) can quickly search the optimal value quickly, the search efficiency is affected by the initialization uniformity, and particles more easily fall into the local optimal solution.

For the above-mentioned defects of particle swarm algorithm, the chaotic motion was applied to the global for better solution, so the initialization process can improve uneven problem of particle swarm, and improve the ability to find the global optimum solution.

The adaptive chaotic particle swarm optimization algorithm which uses integer encoding is adopted in this paper, it can be expressed as $\left\{\mathrm{x}_{1}, x_{2} \ldots x_{2 N_{D G}}\right\}$, the total elements are $2 \times N_{D G}$, where $N_{D G}$ is the number of nodes to be selected for DG. The first $N_{D G}$ elements represent the number of wind turbines installed on each DG that will be selected. The last $N_{D G}$ element represents the number of PV installed on each DG that will be selected. In this paper, in order to improve the convergence of the algorithm and avoid local convergence, in addition to the introduction of chaos optimization theory, the inertia weight and learning factor are improved. There are three main aspects:

1) Chaos algorithm is used to initialization and operation particles. Randomly generated $2 \times N_{D G}$ dimensions, each component of the vector values in the range 0-1 $Z_{1}=\left\{z_{11}, z_{12}, \ldots, z_{12 N_{D G}}\right\}$,According Logistic completely chaotic iterative formula $Z_{n+1}=4 Z_{n}\left(1-Z_{n}\right), n=1,2, \ldots$ Obtained $\mathrm{N}$ vectors $Z_{1}, Z_{2}, \ldots Z_{N}$;By equation $x_{i j}=a_{j}+\left(b_{j}-a_{j}\right) z_{i j}$, (i=1,2,..N; $\mathrm{j}=1,2, \ldots 2 N_{D G}$ ), the vector components to transform the constraints of formula (7); every generation has the $P_{g}$ position vector of chaos optimization, using Logistic iterative equation to generate chaotic sequences, and mapped to the formula (7) constraint range, which are used to calculate each $P_{g}^{m}(m=1,2 \ldots)$ value in the sequence, and to replace the current solution any group of a particle degree.

2) Inertia weight improvements. In order to avoid the fluctuation of the particle in the global solution and improve the search ability of the global solution, the inertia weight can be linearly decreased with the number of iterations, as:

$$
\omega=\omega_{\max }-\frac{\omega_{\text {max }}-\omega_{\min }}{i t e r_{\max }} \times k
$$


where $\omega_{\max }$ is initial weight, $\omega_{\max }$ is final weight, $\omega_{\max }=0.9$, $\omega_{\text {min }}=0.4$, iter ${ }_{\text {max }}$ is the maximum number of iterations, $\mathrm{k}$ is the current iteration number,.

3) Learning factor improvement. In the initial search, the particles should be more learning to their own, less to learn from the best global. Global search ability of particles is reinforced, which avoids falling into local optimum searching early, so $C_{1}$ is bigger value, $C_{2}$ is smaller value. While in the latter part of the search, in order to make the particles closer to the global optimum, and enhance the local search ability, $C_{1}$ is the smaller value, $C_{2}$ is the bigger value,

$$
\begin{gathered}
c_{1}=a+d \cos \left(n \pi / n_{\max }\right) \\
c_{2}=b-d \cos \left(n \pi / n_{\max }\right)
\end{gathered}
$$

if $c_{1}=c_{2}$, then $n=\cos ^{-1}(b-a / 2 d) \times n_{\max } / \pi$. Obviously, when $1 \leq n \leq \cos ^{-1}\left(\frac{b-a}{2 d}\right) \times \frac{n_{\max }}{\pi} \quad$ then $\quad \mathrm{c}_{1}>\mathrm{c}_{2} \quad ; \quad$ and when $\cos ^{-1}\left(\frac{b-a}{2 d}\right) \times \frac{n_{\max }}{\pi} \leq n \leq n_{\max }$, then $\mathrm{c}_{1}<\mathrm{c}_{2}$, where parameters a, b and $\mathrm{d}$ are satisfied, $\mathrm{c}_{1} \in[a-d, a+d]$, $\mathrm{c}_{2} \in[b-d, b+d]$

Considering the time duration, the process of using the improved particle swarm optimization algorithm to solve the DG optimization model of renewable energy sources is as follows:

(1) Algorithm initialization, input and distribution system parameters, such as node parameters, branch parameters, and use chaos algorithm to initialize the population;

(2) Subsequently, the power flow calculation is carried out for each time period to get the fitness value of each particle in the period;

(3) Based on the wind turbine, photovoltaic output and load level during the $t$, the DG installation program represented by each particle is calculated, and the fitness value of each particle in the period of 1 is obtained. If the program does not satisfy the constraint condition of the distribution network, the penalty term is given.;

(4) Let $t=t+1$, and repeat steps (3) to get the adaptation values of next period;

(5) Repeating steps (3) and (4), until all slots are traversed;

(6) After obtaining particle adaptation value of all times, accumulating value of all the particles adaptation period, then the renewable energy utilization of each program is obtained;
(7) Updating both individual extreme and global extreme and updating the particle velocity and position;

(8) Then the optimal particle in this generation population is chaos optimized, and the optimal particle in the chaotic sequence is replaced by any particle in the current population;

(9) Repeat these steps until the termination condition is satisfied, the convergence condition is that the difference between the optimal values of several population generations is smaller than a certain threshold value;

(10) The final output program.

\section{THE ANALYSIS OF EXAMPLES}

In this part, the adaptive chaotic particle swarm optimization algorithm is used to solve the model mentioned above. According to Figure I, three programs have been verified, which are wind power PV combined system, wind power system and photovoltaic system.

\section{A. Parameter Settings}

The network structure of DG is shown as Figure I. Nodes 4, $7,8,14,24,25,29,30,31$ and 32 represent commercial load, the rest of nodes signify resident load. Here its reference voltage is $12.66 \mathrm{kV}$, reference apparent power is 10MVA, the total active load is $3715 \mathrm{~kW}$, and reactive load $2300 \mathrm{kVar}$.

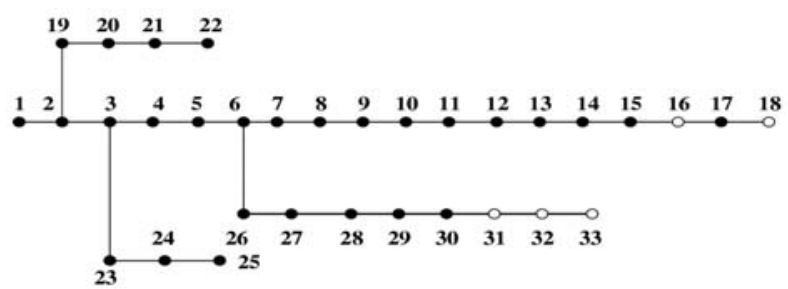

Figure I. 33 nodes distribution system.

Select node 3 16, 18, 31, 32, 33 represented by white dots in Figure I. The type of DG loaded is PV and FAN. Each node voltage and rated voltage deviation does not exceed $7 \%$, $U_{i \min }=0.93, U_{i \max }=1.07$, Line model for overhead line LGJ-70, heat stable transmission limit capacity is 3.86MVA under 40 ${ }^{\circ} \mathrm{C}$; single FAN and PV capacity are $10 \mathrm{~kW}$; max capacity of DG is 200kW; DG total access capacity is $700 \mathrm{~kW}$; adaptive particle swarm optimization algorithm particle population number is 100 , the iteration number is 100 ; Inertia weight $\omega_{\max }=0.9, \omega_{\min }=0.4$, and the number of learning factor is $c_{1}=a+d \cos \left(n \pi / n_{\max }\right), c_{2}=b-d \cos \left(n \pi / n_{\max }\right)$, where a=1.3, $\mathrm{b}=1.2, \mathrm{~d}=1.2$, if $\quad 1 \leq n \leq 0.47 n_{\max } \quad, \quad c_{1}>c_{2} . \quad$ when $0.47 n_{\max } \leq n \leq n_{\max }, c_{1}<c_{2}$.

\section{B. Example Calculations Operators}

By solving the model under three different programs (Program 1: the installation of wind turbine photovoltaic system; Program 2: the installation of wind power; Program 3: the installation of photovoltaic), a planning program will be obtained by implementing the whole process of calculation in 
a typical date of a summer. Then three programs considering time duration are compared with each planning program that consider the time duration, the results are shown in Table I.

It can be seen that the renewable energy utilization in the typical day period is $4.6299 \mathrm{MWh}$ by using installation of FAN and PV cases in program 1, the renewable energy utilization in the typical day period is $4.6299 \mathrm{MWh}$ by using only wind turbine in program 2, and the renewable energy utilization in the typical day period is $4.6737 \mathrm{MWh}$ by using only PV in program3. At the same time, the use of renewable energy capacity of wind turbine and photovoltaic installation is better than individually installed fan and a separate installation of photovoltaic project, the output of wind turbine and the output of photovoltaic have some characteristics of complementary, can achieve stable output in full time range.

In order to analyze the effects of DG for optimal planning results before and after the duration characteristic, this paper first studies the situation without duration characteristic, namely output of wind turbine and photovoltaic is nominal, load is the maximum load. DG optimization planning for three cases ,which include the installation of wind power photovoltaic system (program 4) and the installation of wind power (program 5) and the installation of photovoltaic (program 6), By taking into account the time duration characteristic of these three programs, the node voltage and branch power flow index of each time period are obtained.

The above results obtained are based on a typical situation of the summer days. In order to simulate the reality situation, four typical situations from different seasons are carried out to solve the following DG optimization planning problems, as shown in Table II.

The results show that planning programs are given in above three cases, and at the same time, the installation of wind turbines, photovoltaic renewable energy utilization is higher, reaching 1713.8MWh; and the separate installation of the wind turbine and a separate installation of photovoltaic renewable energy utilization are low, respectively, reached 1689.2MWh and 1685.1MWh. Similarly, the installation of wind turbine and photovoltaic energy utilization at the same time has higher renewable energy utilization than the installation of separate wind turbine and installation of PV. The results verify the feasibility and effectiveness of the model and algorithm for the typical day period of the four seasons.

Table I. Optimal results of planning for DG under time continuous situation by PSO algorithm

\begin{tabular}{ccc}
\hline Program & Program results & $\begin{array}{c}\text { The use of renewable energy } \\
\text { (MWh) }\end{array}$ \\
Program1 & FAN: 8, 8, 1, 6, 18 & 4.6692 \\
Program2 & PV: 0, 20, 2, 5, 2 & 4.6299 \\
& FAN: 13, 16, 9, 12, & 19 \\
Program3 & PV: 20, 9, 0, 20, 20 & 4.6437 \\
\hline
\end{tabular}

Note: The results of the program each digital station number followed by DG node $16,18,31,32,33$ loaded.
Table II. Optimal results of planning for DG under annual time continuous by PSO algorithm

\begin{tabular}{ccc}
\hline Program & Program Results & $\begin{array}{c}\text { The use of renewable } \\
\text { energy (MWh) }\end{array}$ \\
Program 4 & Fan: 6, 4, 20, 13, 20 & 1713.8 \\
Program 5 & PV: 0, 6, 0, 0, 0 & 1689.2 \\
Program 6 & 6, 20, 13, 20, 14, 19 & 1685.1 \\
\hline
\end{tabular}

Note: The results of the program each digital station number followed by DG node $16,18,31,32,33$ loaded.

\section{CONCLUSIONS}

Based on considering DG output and load characteristics of duration time, a planning model aiming at maximum utilization of renewable energy is discussed in this paper. And an adaptive chaos particle swarm optimization is employed to solve this optimization model. A DG planning schedule is obtained to meet constraints during all time period, and installation sites and capacities are given to guarantee the maximum utilization of the renewable energy DG in the premise of system safe and stability, which could relieve environmental pollution and resource crisis.

\section{ACKNOWLEDGMENT}

This work is supported by State Grid Hubei Electronic Power Company of China under Grant No. 52153814000D.

\section{REFERENCES}

[1] F. Mingtian, Z. Zuping, S. Aoxue, and S. Jian, "Enabling Technologies for Active Distribution Systems,” J Proceedings of the CSEE, 2013, vol. 33(22), pp.12-18.

[2] S. Alavi, A. Ahmadian, and M. Aliakbar-Golkar, "Optimal probabilistic energy management in a typical micro-grid based on robust optimization and point estimate method,” Energy Convers Manage, 2015, vol. 95, pp. 314-325.

[3] Z. Feifei, L. Zongqi, G. Guoliang, "Research on Power Saturated Grid Planning Method by Considering of the Influence of Years on the Power Grid in Close-range Years,” J, Modern Electric Power,2012, vol. 29(3), pp. 12-16.

[4] F. Chen, Z. Xiang, C. Haozhong, Z. Shenxi, and Y. Liangzhong. "Framework Planning of Distribution Network Containing Distributed Generation Considering Active Management,” Power System Technology, 2014, vol. 38(4), pp. 823-829.

[5] C. Haihua, Z. Yaxian, G. Jian, "Path Optimization Model of Trans-regional and Trans-provincial Electricity Trade Based on Expand Network Flow," J.Automation of Electric Power Systems, 2007, vol. 31(17), pp.21-25.

[6] A. Zangeneh, S. Jadid, "Fuzzy multi-objective model for distributed generation expansion planning in uncertain environment," European Transactions on Electrical Power, 2011, vol. 21(1), pp. 129-141.

[7] Y. Yi, W. Gang, Z. Bing, Z. Xin, "Optimized Fuzzy Planning of the Distribution Network Including Distributed Generation,” Automation of Electric Power Systems, 2010, vol. 34(13), pp.19-23. 